\title{
Enfermedad asociada a Clostridium difficile (EACD) en el Hospital Escuela Universitario y el IHSS. Enero a mayo 2016
}

Miriam Esther Arias, Edgardo Tzoc, Fanny Hidalgo

\section{RESUMEN}

Clostridium difficile es una bacteria Gram positiva anaerobia, productora de esporas responsable de la diarrea asociada al uso de antibióticos, en últimos años se han reportado brotes dentro de hospitales a nivel mundial. La enfermedad asociada a $C$. difficile (EACD) puede manifestarse de diferentes formas, entre las que destaca una inflamación del colon que se manifiesta como un cuadro diarreico que aparece frecuentemente tras el uso de antimicrobianos, especialmente clindamicina, cefalosporinas y fluoroquinolonas. Durante los meses de enero a mayo de 2016 se realizó un estudio descriptivo en los hospitales Escuela Universitario e IHSS. El objetivo general del estudio fue analizar las características microbiológicas y epidemiológicas de la enfermedad asociada a Clostridium difficile en pacientes con sospecha clínica en Instituto Hondureño de Seguridad Social y Hospital Escuela Universitario, Tegucigalpa, enero-mayo 2016 de ambos hospitales. El análisis microbiológico consistió en la detección de las toxinas A y $B$ de la bacteria mediante una prueba serológica de inmunocromatografía y mediante el aislamiento por cultivo anaerobio. El análisis epidemiológico se hizo mediante la recolección de información de los pacientes en fichas epidemiológicas, previamente se obtuvo el consentimiento informado de cada paciente. Los principales resultados obtenidos fueron una prevalencia puntual para el periodo de $237 \%$ de acuerdo con la prueba diagnóstica y los factores de riesgo asociados más frecuentes fueron la edad avanzada, un promedio de edad de los pacientes fue de 55 años y el $41 \%$ de los pacientes confirmados fueron de la tercera edad. Asimismo, el amplio uso de antimicrobianos como la clindamicina y las cefalosporinas en 33 y $60 \%$ respectivamente se manifiesta como un factor decisivo para el desarrollo de las EACD. En conclusión, $C$. difficile es una bacteria que se encuentra

\footnotetext{
${ }^{1}$ Beneficiarios de una beca básica de la DICYP. Grupo de Investigación en Enfermedades de Etiología Bacteriana. Profesores de la sección de Bacteriología, Departamento de Microbiología, Facultad de Ciencias, UNAH: arias_miriam2004@yahoo.com; edgardo.ramirez@unah.edu.hn; fanny_hidalgo@hotmail.com
} 
en los principales centros asistenciales de nuestro país, su asociación como agente causal de infecciones y enfermedad intrahospitalaria está bien establecida. La ocurrencia de la enfermedad se relaciona con las condiciones de infraestructura que facilitan su colonización y transmisión.

Palabras clave: Clostridium difficile, diarrea nosocomial, diarrea por antibióticos, toxinas $A$ y $B$.

\section{ABSTRACT}

Clostridium difficile is an anaerobic, spore-producing Gram positive bacterium responsible for the diarrhea associated with the use of antibiotics, in recent years outbreaks have been reported in hospitals worldwide. The disease associated with $C$. difficile (CDAD) can manifest itself in different ways, among which is an inflammation of the colon that manifests as a diarrheal disease that frequently appears after the use of antimicrobials, especially clindamycin, cephalosporins and fluoroquinolones. During the months of January to May 2016, a descriptive study was carried out in HEU and IHSS hospitals. The general objective of the study was to analyze the microbiological and epidemiological characteristics of the disease associated with Clostridium difficile CDAD in patients with clinical suspicion in the Honduran HEU and IHSS, Tegucigalpa, January-May 2016 of both hospitals. The microbiological analysis consisted in the detection of toxins $A$ and $B$ of the bacteria by means of a serological test of immunochromatography and by isolation by anaerobic culture. The epidemiological analysis was done by collecting information from patients in epidemiological files, previously the informed consent of each patient was obtained. The main results obtained were a point prevalence for the period of $237 \%$ according to the diagnostic test and the most frequent associated risk factors were advanced age, an average age of the patients of 55 years and $41 \%$ of the patients. Confirmed patients were elderly. Likewise, the widespread use of antimicrobials such as clindamycin and cephalosporins in 33\% and $60 \%$ respectively is manifested as a decisive factor for the development of CDAD. In conclusion, $C$. difficile is a bacterium found in the main healthcare centers of our country and its association as a causative agent of infections and intrahospital disease is well established. The occurrence of the disease is related to the infrastructure conditions that facilitate its colonization and transmission.

Key words: Clostridium difficile, nosocomial diarrhea, diarrhea by antibiotics, toxins $A$ and $B$. 


\section{INTRODUCCIÓN}

C. difficile es una bacteria Gram positiva anaerobia, productora de esporas responsable de un porcentaje importante de diarrea asociada al uso de antibióticos de amplio espectro en el ámbito hospitalario. Fue identificado en 1935 como Bacillus difficilis en la flora fecal de niños sanos y reconocido como causa de la colitis asociada a antibióticos (Lopardo et al. 2015; Price, Larson, and Crow 1979).

En algunos países se le refiere como el responsable de $25-30 \%$ de todas las diarreas adquiridas intrahospitalariamente (Freeman et al. 2010; Rodríguez-Pardo, Mirelis, and Navarro 2013). Además de su asociación con las diarreas asociadas a antimicrobianos es reconocido como causa de casos graves de colitis pseudomembranosa y megacolon tóxico (Barra-carrasco et al. 2014), manifestaciones clínicas importantes en el ámbito hospitalario.

Varios factores son requeridos para que $C$. difficile cause enfermedad. El primero y más importante es la alteración de la microbiota intestinal normal, generalmente por el uso de antibióticos, especialmente clindamicina, penicilinas, cefalosporinas y recientemente fluoroquinolonas, aunque prácticamente todos los antibióticos tienen el potencial de predisponer a esta infección (Van Kleef et al. 2014). El uso extendido de antibióticos se ha asociado a un aumento de resistencia a antimicrobianos y constituye el factor de riesgo más importante para el desarrollo de diarrea asociada a C. difficile (Amy et al. 2015).

Aproximadamente $3 \%$ de los adultos colonizados son asintomáticos, en áreas de hospitalización son colonizados de 20 a 30\% de los pacientes y en unidades de cuidado crónico hasta 50\% (Knecht et al. 2014).

Clostridium difficile es responsable de $15-20 \%$ de los casos de diarrea relacionados con antibióticos y casi la totalidad de los casos de colitis pseudomembranosa(Amy et al. 2015). En pacientes de la tercera edad hospitalizados, la incidencia de Infección por $C$. difficile es 5 a 10 veces más alta comparada con los pacientes jóvenes. La mortalidad global asociada con diarrea por $C$. difficile infecciosa se estima en $17 \%$ en brotes dentro de los hospitales; mientras que la tasa de adquisición de C. difficile estimada es de $13 \%$ en los pacientes con estancias hospitalarias de 2 semanas y $50 \%$ en aquellos con estancias superiores a 4 semanas(Cohen, Gerding, and Johnson 2010; Siller-Ruiz et al. 2014). 
Por lo anteriormente escrito, surgió la necesidad de investigar la frecuencia de las EACD en centros hospitalarios de mayor demanda en el país, ya que en la actualidad, en Honduras la situación con respecto a la ocurrencia de las infecciones causadas por esta bacteria es desconocida casi por completo. Se sabe que las infecciones se presentan en pacientes hospitalizados, por la descripción clínica, pero nunca se ha establecido un diagnóstico preciso basado en la evidencia de las pruebas de laboratorio.

Por otro lado, existe un problema de diagnóstico de las diarreas causadas por C. difficile, debido a la dificultad para la confirmación de laboratorio que exige la identificación y la demostración de toxina de la bacteria; y debido a que esta es una bacteria fastidiosa, anaerobia y de difícil aislamiento, los laboratorios no cuentan con la capacidad para hacer un diagnóstico oportuno, por lo que los médicos no tienen alternativa sino dar tratamiento empírico al paciente guiándose solamente por la clínica, sin tener confirmación del laboratorio.

En Honduras no se ha realizado ningún estudio relacionado al tema por lo tanto no existen datos sobre la prevalencia de diarreas causadas por $C$. difficile, en particular por lo que este estudio es un primer aporte sobre esta problemática de salud pública a nivel mundial y que está siendo cada vez más estudiada por la extensión de las complicaciones que causa debido a la falta de un diagnóstico oportuno. Por otra parte, el conocimiento preciso de los principales factores de riesgo asociados a la ocurrencia de estas infecciones en los hospitales de nuestro país posibilita la implementación de las medidas pertinentes para la mitigación del impacto causado por este patógeno, siendo que es conocido que cada centro de atención en salud presenta sus propias particularidades que influyen en la mayor o menor extensión del problema.

\section{Participantes y Métodos}

Diseño. Esta es una investigación aplicada dentro del campo de la microbiología clínica, siendo un estudio descriptivo transversal. El objetivo fue describir las características de Clostridium difficile como agente causal de diarrea limitada al ámbito hospitalario en dos de los principales centros de atención en salud de la ciudad de Tegucigalpa, el Hospital Escuela Universitario y el Hospital de Especialidades del Instituto Hondureño del Seguro Social. La duración del estudio fue de cinco meses, de enero a mayo de 2016.

Entorno. Los hospitales donde se realizó el estudio fueron seleccionados debido a 
que son los más grandes del sector público en la zona central del país. Ambos ofrecen la mayor cantidad de especialidades médicas y servicios de hospitalización. El Hospital Escuela Universitario se encuentra ubicado en la ciudad de Tegucigalpa y el Hospital de especialidades del IHSS se encuentra ubicado en la ciudad de Comayagüela.

\section{Participantes}

La población en estudio fueron pacientes que cumplieron con los criterios de inclusión, hospitalizados en el Instituto Hondureño de Seguridad Social y el Hospital Escuela Universitario, Tegucigalpa, Honduras durante el período de estudio y que además aceptaron participar en el estudio firmando el consentimiento 0 asentimiento informado previo.

\section{Criterio de inclusión}

Persona de cualquier edad, hombre o mujer; hospitalizado en el Instituto Hondureño de Seguridad Social y el Hospital Escuela Universitario, con presencia de diarrea definida como 3 o más evacuaciones de heces no formadas en 24 horas consecutivas o menos, que el motivo de ingreso de la persona al hospital no sea por diarrea, que la diarrea se presente $48 \mathrm{~h}$ después de haber ingresado al hospital y que el médico a cargo del paciente avale la sospecha clínica de una EACD.

Una vez firmado el consentimiento informado, se procedió a aplicar el cuestionario clínico, también se le explicó al paciente el procedimiento de la toma de la muestra. Este procedimiento fue realizado por estudiantes de la carrera de Microbiología capacitados para tal fin.

Muestras. El muestreo utilizado fue de tipo no probabilístico, no aleatorio. Todas las muestras recolectadas en período de tiempo establecido: enero a mayo de 2016. Estas fueron recolectadas por personal de los hospitales y almacenadas en congelación a $-20^{\circ} \mathrm{C}$ hasta su traslado al laboratorio de Bacteriología de la Escuela de Microbiología de la UNAH, donde se realizaron los ensayos de laboratorio. El transporte de las muestras se hizo respetando la cadena de frio a $4{ }^{\circ} \mathrm{C}$.

Aspectos éticos: El presente estudio no representó ningún riesgo para los participantes, siguiéndose un riguroso proceso de codificación de las muestras para la protección de la privacidad y confidencialidad de los individuos. El protocolo de investigación de este estudio fue evaluado por el Comité de Ética de Investigación de la Maes- 
tría de Enfermedades Infecciosas y Zoonóticas (CEI-MEIZ).

Implementación del estudio: El presente estudio se llevó a cabo en el IHSS y HEU, cuando el médico tuvo una sospecha clínica de un paciente con ICD, se procedió a informar al responsable de la investigación en cada uno de los centros asistenciales, el cual explicó el consentimiento informado al paciente, y este procedió a firmarlo. Una vez firmado el consentimiento informado, se procedió a aplicar el cuestionario clínico y también se le explicó al personal médico y de enfermería las condiciones de la toma de la muestra de heces. La muestra fue trasladada inmediatamente en una hielera al Laboratorio de Bacteriología (UNAH), donde se realizaron las primeras pruebas, como la detección de toxinas y cultivo anaerobio. A continuación, se describe a detalle el proceso antes mencionado.

\section{Instrumentos y técnicas}

1. Procesamiento de muestras fecales:

Detección de toxinas $A$ y $B$ de $C$. difficile: Se realizó utilizando la prueba Xpect $C$. difficile $A / B$ test de Oxoid $\cap$ siguiendo las instrucciones del fabricante.

Cultivo anaerobio de C. difficile: Se utilizó el método descrito por Rodríguez-Palacios y colaboradores del Colegio Veterinario de Ontario, Canadá, con la modificaciones hechas por Quesada Gómez (Quesada-Gómez et al. 2015) del Laboratorio de Investigación en Bacterias Anaerobias (LIBA) de la Facultad de Microbiología de la Universidad de Costa Rica.

Brevemente: En tubos cónicos se dispensaron $0.5 \mathrm{~mL}$ de solución estéril de extracto de levadura y se agregó igual volumen de muestra. Se adicionaron $1 \mathrm{~mL}$ de etanol, se mezcló en vortex y se incubó a temperatura ambiente por 50 minutos. Luego se centrifugó a 3800 rpm por 10 minutos. Se decantó e inoculó el sedimento con hisopo en placas de agar CCFA. Luego se incubó en jarra de anaerobiosis por 48 horas y se examinaron las placas en busca de colonias típicas color amarillo.

Alternativamente y como respaldo, se introdujo el hisopo usado para inocular las placas de agar en tubos de caldo FAB y se incubaron por 7 días. Si las placas de agar CCFA resultaron positivas, estos se descartaron. Si resultaron negativas, después de la incubación de 7 días se inocularon en placas de agar CCFA e incubaron en condiciones apropiadas. 
Las colonias sospechosas se subcultivan en agar BAK. Se revisaron las placas por pureza. Se verificó la morfología celular con tinción de Gram y fluorescencia con luz UV. Por último se hizo la prueba de aerotolerancia. Las colonias presuntivamente identificadas como C. difficile se sometieron a Identificación con el sistema Rapid ANA II Panel de Oxoid®.

Los aislamientos identificados como $\mathrm{C}$. difficile se guardaron a $-20^{\circ} \mathrm{C}$ para su posterior caracterización molecular.

2. Recolección de información epidemiológica. El levantamiento de la información epidemiológica se hizo mediante el llenado de una ficha diseñada y validada previamente.

3. Plan de análisis. Para el análisis de los resultados se obtuvo la frecuencia de cada uno de los datos, utilizando el programa estadístico SPSS para el análisis de a estadística descriptiva de los análisis microbiológicos y de la información epidemiológica obtenida de la revisión de expedientes.

\section{RESULTADOS}

Durante el período comprendido de enero a junio del año 2016, se analizaron un total de 38 muestras fecales provenientes de pacientes hospitalizados en el Hospital Escuela Universitario $(n=26)$ y el hospital del Instituto Hondureño de Seguridad Social $(n=12)$.

El $60 \%$ fueron mujeres, y la distribución por edad muestra proporciones similares entre los grupos etarios; $32 \%$ para pacientes pediátricos, para adultos mayores y el resto para población adulta. Las EACD se asocian a la adquisición intrahospitalaria, por lo cual el motivo de ingreso al hospital debe ser uno diferente a la infección gastrointestinal manifiesta con diarrea.

Se describe la frecuencia de las principales condiciones subyacentes presentadas en los pacientes y que explican el motivo de su hospitalización. En los pacientes menores de edad la principal causa es la malignidad con un 75\% (Cuadro 1). Esta se manifiesta por diversos tipos de cáncer, entre estos son particularmente frecuentes las leucemias. 
Por otra parte, en los adultos las principales patologías de base son las denominadas enfermedades metabólicas como la diabetes con un $19 \%$ y otras afecciones gastrointestinales con un $31 \%$. Los traumas como fracturas, las infecciones del tracto respiratorio bajo como la neumonía y las infecciones generalizadas que llevan a un estado denominado sepsis representan porcentajes mucho menores de entre 3 y $5 \%$. Es de hacer notar que en un $29 \%$ del total de los pacientes no fue posible obtener la información con respecto a esta variable.

Cuadro 1. Diagnóstico inicial de ingreso de los pacientes incluidos en el estudio. Hospital Escuela Universitarios e IHSS. Enero a junio de 2016.

\begin{tabular}{|c|c|c|}
\hline Diagnóstico de inicio & Menores de edad & Adultos \\
\hline Malignidad & 9 & 0 \\
\hline Sepsis & 1 & 2 \\
\hline Trauma & 0 & 1 \\
\hline Diabetes & 0 & 5 \\
\hline Respiratorio & 1 & 0 \\
\hline Gastrointestinal & 0 & 8 \\
\hline ND & 1 & 10 \\
\hline
\end{tabular}

*La información se obtuvo a partir del cuestionario clínico - epidemiológico.

Las Cuadros 2 y 3 presentan el empleo de diversos medicamentos que podrían influir en la adquisición del agente etiológico y el desarrollo de la infección y la enfermedad. De estos, los antibióticos son los principales medicamentos asociados a la aparición de las EACD. De acuerdo a la tabla 2 en un $83 \%$ de los pacientes se utilizó una terapia antibacteriana antes de la aparición de los síntomas típicos de la enfermedad. Los antimicóticos y los antiparasitarios empleados en solo un $6 \%$ no se consideran ya que tienen poco sino ningún efecto asociado a la enfermedad. Los antiinflamatorios no esteroideos, los inhibidores de la bomba de protones y los corticosteroides empleados en un 6 a $11 \%$ si son medicamentos que en mayor o menor grado pueden incidir en la ocurrencia de la enfermedad al facilitar la colonización por Clostridium difficile. 
Cuadro 2. Medicamentos empleados previamente en los pacientes, previo a su ingreso al hospital. Hospital Escuela Universitarios e IHSS. Enero a junio de 2016.

\begin{tabular}{|c|c|}
\hline & Porcentaje de uso \\
\hline \multicolumn{2}{|l|}{ Tipo de medicamento empleado } \\
\hline Diuréticos & 6 \\
\hline Inhibidores de la bomba de protones & 11 \\
\hline Antiinflamatorios no esteroideos & 6 \\
\hline Corticosteroides & 11 \\
\hline Antibióticos & 83 \\
\hline Antimicóticos & 6 \\
\hline Antiparasitarios & 6 \\
\hline \multicolumn{2}{|l|}{ Empleo de antibióticos } \\
\hline Al menos un antibiótico & 44 \\
\hline Al menos dos antibióticos & 22 \\
\hline Tres o más antibióticos & 34 \\
\hline
\end{tabular}

*La información se obtuvo a partir del cuestionario clínico - epidemiológico.

Con respecto a la frecuencia de uso de los antibióticos, la tabla 3 indica que del 100\% de los pacientes que tuvieron una terapia antimicrobiana la mayor estrategia de uso fue con un solo medicamento representando un $44 \%$. Muy significativo es el hecho que la terapia combinada con dos o más antibióticos representan el $56 \%$ de los pacientes tratados. Estos son datos sumamente interesantes ya que teóricamente el uso de al menos un solo antibiótico es un factor de riesgo, el cual incrementa en tanto se eleva el número de antibióticos en terapias combinadas.

De acuerdo a los diferentes grupos de antibióticos utilizados previamente, los más empleados como era de esperarse pertenecen al grupo de las cefalosporinas y de las penicilinas, ambos Beta lactámicos. En conjunto representan un 100\% de uso entre los pacientes. No se pudo determinar los antibióticos empleados pero los más comunes empleados en el ámbito comunitario y hospitalario son las penicilinas naturales, las aminopeniclinas y las cefalosporinas de segunda y tercera generación. También 
en la tabla 4 se observa que la clindamicina, perteneciente al grupo de las lincosamidas, representa un tercio de los antibióticos en uso.

Esto es un dato que resalta puesto que este antibiótico es el más comúnmente asociado a la aparición de casos de EACD y por su manera de administración por vía parenteral se restringe en gran medida al uso dentro de los hospitales, aunque también se utiliza en presentaciones para uso por vía oral. En menor porcentaje se observan antibióticos pertenecientes también a grupos restringidos al ámbito intrahospitalario como los aminoglicósidos, los glicopéptidos y las quinolonas.

Cuadro 3. Grupos de antibióticos empleados previamente en los pacientes, previo a su ingreso al hospital. Enero a junio de 2016.

\begin{tabular}{|l|c|}
\hline \multicolumn{1}{|c|}{ Grupo de antibióticos } & Porcentaje (\%) \\
\hline Lincosamidas (clindamicina) & 33 \\
\hline Cefalosporinas & 60 \\
\hline Carbapenems & 27 \\
\hline Penicilinas & 40 \\
\hline Aminoglicósidos & 13 \\
\hline Fluoroquinolonas & 7 \\
\hline Glicopéptidos & 7 \\
\hline
\end{tabular}

*La información se obtuvo a partir del cuestionario clínico - epidemiológico.

Se obtuvo una muestra de heces de cada uno de los 38 pacientes incluidos en el estudio. Estas se sometieron a análisis por dos métodos: detección de las toxinas Ay $B$ por medio de un kit serológico comercial de inmunocromatografía y por cultivo después de un shock con etanol en medio selectivo CCFA. El cuadro 4 nos indica los porcentajes de positividad para ambos métodos. Del total de 38 muestras se obtuvo un mayor porcentaje mediante técnicas de cultivo que de inmunocromatografía.

Por hospitales, la tabla 6 muestra un porcentaje similar para ambos hospitales cuando se suman los resultados por ambos métodos. Inclusive muestran resultados comparables con el método diagnóstico serológico (23 y $25 \%$ ), resultando diferente únicamente por el método de cultivo obteniéndose un porcentaje bastante mayor para el HEU. 
Cuadro 4. Resultados de análisis de muestras por tipo de ensayo en muestras tomadas del Hospital Escuela Universitario e IHSS. Enero a junio de 2016.

\begin{tabular}{|l|c|c|c|}
\hline \multirow{2}{*}{} & $\mathbf{1 \%}$ & \multicolumn{2}{|c|}{ Porcentaje por Hospital } \\
\cline { 2 - 4 } & Positivos $(\mathbf{n}=\mathbf{3 8})$ & HEU ( $\mathbf{n}=\mathbf{2 6})$ & IHSS ( $\mathbf{= 1 2})$ \\
\hline Detección de toxinas & 23.7 & 23 & 25 \\
\hline Cultivo de heces & 34.2 & 38 & 25 \\
\hline
\end{tabular}

*La detección de las toxinas se realizó utilizando la prueba Xpect C. difficile A/B test de Oxoid ${ }^{\circledR}$ *El cultivo anaerobio se realizó en CCFA y BAK.

\section{DISCUSIÓN}

En Honduras, el estudio de las EACD no se hace de rutina en los hospitales públicos, únicamente a solicitud del médico y debido a una fuerte sospecha clínica es que se pide a los familiares del paciente el envío de una muestra a un laboratorio privado para su análisis. Hasta el momento, esta es la primera investigación sobre las infecciones causada por $C$. difficile en el ámbito hospitalario de nuestro país.

En el presente estudio se determinó una prevalencia puntual para el periodo comprendido entre enero a junio de 2016 del $23.7 \%$ de diarrea por Clostridium difficile para las diarreas nosocomiales con diagnóstico inicial de EACD en el hospital Escuela Universitario y el hospital de especialidades del Instituto Hondureño del Seguro Social IHSS. Este resultado se encuentra muy próximo a lo reportado previamente en otros países de entre 20 y 30\% (Pérez et al. 2013) y 15-25\% (Cohen, Gerding, and Johnson 2010). Sin embargo, otros estudios reportan prevalencias puntuales significativamente más altas en condiciones similares de criterios de inclusión(He et al. 2012; Kuijper, Coignard, and Tu 2006).

La población tan heterogénea incluida en este estudio explica en parte los resultados obtenidos. A diferencia de otros donde la población se delimita por rangos de edad, en este estudio se han incluido personas de cualquier edad. Cuando la población es predominantemente pediátrica se reportan datos de prevalencia o incidencia más bajos a diferencia de los resultados obtenidos en hospitales donde se atienden exclusivamente a personas adultas y donde se concentra una mayor proporción de población geriátrica. Las razones por las cuales hay una menor prevalencia e incidencia en 
niños no está aún bien establecida, pero se conoce que la colonización en niños menores de un año es bastante más alta que en adultos y a partir de los dos años se acerca a las tasas comunes en adultos (Rodríguez C, 2016,Cd infection, molecular strain typing methods n.d.; Village 2013). Inclusive, aun en presencia de cepas toxigenicas, el desarrollo de EACD no es tan evidente en niños muy probablemente debido a características biológicas intrínsecas en los niños que no se encuentran en los adultos.

La edad es uno de los principales factores de riesgo de las EACD siendo las personas mayores de 65 años especialmente susceptibles a estas. En este estudio, el $33 \%$ de las personas con diagnóstico inicial pertenecian a este segmento de la población (entre un promedio general de 55 años), y el $41 \%$ de los casos confirmados por laboratorio fueron adultos mayores. Este aspecto concuerda con lo reportado en brotes descritos en países de la región centroamericana donde la mayoría de afectados eran de la población adulta mayor (Amy et al. 2015).

Por el contrario, los casos confirmados pertenecientes a menores de edad apenas representan el $6 \%$ del total aun y cuando la población pediátrica representó la misma proporción que los adultos mayores. Porqué esta población se convierte en los principales afectados probablemente se debe a la mayor cantidad de condiciones subyacentes potencialmente predisponentes y su estado inmunológico naturalmente deprimido.

Entre la variedad de factores externos al paciente, el uso de medicamentos es el más importante. Se mencionan entre otros, los agentes quimioterapéuticos, los inhibidores de la bomba de protones y los antimicrobianos. Los resultados muestran que una parte importante de la población en estudio diagnosticada inicialmente con EACD estaba bajo una terapia con agentes anticancerígenos en un 23\% (niños hospitalizados en sala de oncología pediátrica). Pero la terapia con inhibidores de la bomba de protones apenas representó un $11 \%$. Ambos medicamentos son reconocidos en la literatura como un factor extrínseco altamente relacionado con el desarrollo de la enfermedad (Goudarzi et al. 2014; Microbiota and Villalobos 2014) con respecto a estos últimos no existe unanimidad de criterios con respecto a si en efecto son predisponentes ya que no se conoce muy bien el mecanismo por el cual podrían contribuir a este fenómeno(Barra-carrasco et al. 2014; Pérez et al. 2013).

Con respecto al uso de antimicrobianos nuestros resultados coinciden con los reportados en diversos países del mundo y que en la actualidad son reconocidos como los más frecuentemente asociados al problema. De hecho, el uso de antimicrobianos al 
menos con 14 días de antelación y hasta 3 meses es uno de los factores comunes en el $100 \%$ de los pacientes sintomáticos (Cohen, Gerding, and Johnson 2010). Aunque en teoría cualquier antibiótico puede ser relacionado con la enfermedad, medicamentos como la clindamicina y las cefalosporinas identificadas en proporción de uso de 33 y $60 \%$ respectivamente explican en parte las tasas de prevalencia para las EACD en los hospitales en estudio al igual que lo reportado por otros investigadores (Amy et al. 2015; Lopardo et al. 2015).

El tratamiento de los pacientes con diagnostico preliminar de EACD en base a las características clínicas recibió el tratamiento indicado que es primariamente con metronidazol en un $53 \%$ (solo o en terapias combinadas) y de $54 \%$ para el tratamiento con vancomicina (solo o en terapias combinadas) lo cual difiere con lo reportado en hospitales de otros países (Camacho-Ortiz et al. 2015).

\section{CONCLUSIONES}

La prevalencia obtenida, similar a lo reportado en estudios de todo el mundo, evidencia las condiciones presentes en el HEU e IHSS con respecto a los factores de riesgo asociados; la edad avanzada, las enfermedades subyacentes, el amplio uso de medicamentos predisponentes y el estado nutricional fueron los más frecuentemente reportados. La población más vulnerable resultó ser la de personas mayores de 60 años, ya que en estos se dan las condiciones ideales para que la bacteria logre su desarrollo al grado de presentarse las formas más severas de la enfermedad con tasas de mortalidad más significativas. Sin embargo, la población menor de edad con diagnósticos de enfermedades malignas también es de especial atención con respecto al desarrollo de la EACD.

El uso masivo e indiscriminado de antimicrobianos en los hospitales nacionales resultó ser uno de los principales factores asociados al desarrollo de las EACD. Esto debido no solo al uso de estos medicamentos para el tratamiento de infecciones sino también por su uso como profiláctico aun cuando se desconoce la verdadera etiología de las infecciones intrahospitalarias. Este es un hecho que se aplica no solo a este tema en particular sino al ámbito de las infecciones nosocomiales de etiología bacteriana. 


\section{AGRADECIMIENTOS}

La realización de este estudio fue posible gracias al aporte económico de la Dirección de Investigación Científica de la UNAH mediante beca básica de investigación otorgada en el año 2015. Expresamos especial gratitud al comité de enfermedades infecciosas del IHSS y HEU, a los laboratorios de ambos centros hospitalarios por su colaboración en la conservación de las muestras. También agradecemos a Sarhy Guevara, Cinthia Mendoza y Héctor Ventura, estudiantes de la Escuela de Microbiología, por su valiosa contribución en la recolección de las muestras, cultivo bacteriano, recolección y manejo de la información epidemiológica.

\section{REFERENCIAS BIBLIOGRÁFICAS}

Amy, Jacob M. et al. (2015). "Clostridium Difficile Infection." PLOS ONE 10(1): 1-9. http://www.tandfonline.com/doi/full/10.4161/21645515.2014.980193.

Barra-carrasco, Jonathan et al. (2014). "Esporas De." 31(6): 694-703.

Camacho-Ortiz, Adrián et al. (2015). "First Report of Clostridium Difficile NAP1/027 in a Mexican Hospital." PLoS ONE 10(4): 1-11.

Cohen, SH, DN Gerding, and S Johnson. 2010. "Clinical Practice Guidelines for Clostridium Difficile Infection in Adults: (2010) Update by the Society for Healthcare Epidemiology of America (SHEA) and the Infectious." Infection Control \&. http://journals.cambridge.org/abstract_S0195941700029064 (November 4, 2016).

Freeman, J. et al. (2010). "The Changing Epidemiology of Clostridium Difficile Infections." Clinical Microbiology Reviews 23(3): 529-49.

Goudarzi, Mehdi et al. (2014). "Clostridium Difficile Infection: Epidemiology, Pathogenesis, Risk Factors, and Therapeutic Options." Scientifica 2014: 1-9. http://www.hindawi.com/journals/scientifica/2014/916826/.

$\mathrm{He}$, Miao et al. (2012). "Europe PMC Funders Group Emergence and Global Spread of Epidemic Healthcare-Associated Clostridium Difficile." 45(1): 109-13.

Van Kleef, Esther et al. (2014). "Nosocomial Transmission of c. Difficile in English Hospitals from Patients with Symptomatic Infection." PLOS ONE 9(6).

Knecht, Henrik et al. (2014). "Effects of $\otimes$ ??-Lactam Antibiotics and Fluoroquinolones on Human Gut Microbiota in Relation to Clostridium Difficile Associated Diarrhea." PloS one $9(2)$.

Kuijper, E J, B Coignard, and P Tu. (2006). "Emergence of Clostridium Difficile -Associated Disease in North America and Europe." Clinical Microbiology and Infections 12(6): 2-18.

Lopardo, Gustavo et al. (2015). "Epidemiology of Clostridium Difficile: A Hospital-Based Descriptive Study in Argentina and Mexico." Brazilian Journal of Infectious Diseases 
19(1): 8-14. http://dx.doi.org/10.1016/j.bjid.2014.07.004.

Microbiota, Gastrotrilogía I, and Gonzalo Torres Villalobos. (2014). Dr. Miguel Ángel Valdovinos Díaz M . C . Elisa Gómez Reyes.

Pérez, ÁB, ÓR Morales, WO Regino, and MG Zuleta. (2013). "Clostridium Difficile Infections in Elderly Patients." Rev Col Gastroenterol 28(1): 53-63. http://www.gastrocol.com/file/Revista/en_v28n1a07.pdf.

Price, a B, H E Larson, and J Crow. (1979). "Morphology of Experimental Antibiotic-Associated Enterocolitis in the Hamster: A Model for Human Pseudomembranous Colitis and Antibiotic-Associated Diarrhoea." Gut 20(6): 467-75.

Quesada-Gómez, Carlos et al. (2015). "Emergence of an Outbreak-Associated Clostridium Difficile Variant with Increased Virulence." Journal of Clinical Microbiology 53(4): 1216-26.

Rodríguez-Pardo, Dolors, Beatriz Mirelis, and Ferran Navarro. (2013). "Infecciones Producidas Por Clostridium Difficile." Enfermedades Infecciosas y Microbiologia Clinica 31(4): 254-63. http://dx.doi.org/10.1016/j.eimc.2012.12.010.

"Rodríguez C, 2016,Cd Infection, Molecular Strain Typing Methods."

Siller-Ruiz, María et al. (2014). "Epidemiolog de La Enfermedad Asociada a Clostridium Difficile (EACD) En Salamanca." Revista Espanola de Quimioterapia 27(2): 122-26.

Village, Elk Grove. (2013). "Clostridium Difficile Infection in Infants and Children." Pediatrics 131(1): 196-200. http://www.ncbi.nlm.nih.gov/pubmed/23277317. 